\title{
The role of intrastromal voriconazole in deep resistant fungal keratitis
}

\author{
Punit K. Singh \\ Medical College, Jodhpur, India
}

\begin{abstract}
Targeted delivery of voriconazole (azole) by intrastromal perilesional injection may be a new safe and effective way to treat cases of deep-seated challenging fungal keratitis responding poorly to conventional treatment.
\end{abstract}

Key words: Voriconazole, intrastromal, fungal keratitis

\section{Introduction}

Voriconazole has been reported to be effective in the treatment of fungal keratitis. Voriconazole has the broad spectrum coverage of the azole antifungals and has good intraocular penetration following oral administration. Fungal corneal infections always pose a challenge for the ophthalmologists. Modern antifungal drugs in the treatment of mycotic keratitis are less effective than contemporary antibacterial drugs in the treatment of bacterial keratitis. The penetration of many antifungal drugs into the cornea is inadeqate, which makes it challenging to treat cases of deep fungal keratitis. To overcome these problems, investigators have evaluated alternate routes such as intracameral and intrastromal injections to treat fungal keratitis. ${ }^{1-3}$ Recently, there have been reports of effectiveness of the newer azole drug Voriconazole by oral and local targeted drug delivery routes by intrastromal injections..$^{4-6}$ We here report two cases in which intrastromal voriconazole was used with topical treatment to treat deep-seated, resistant, fungal keratitis.

\section{Case report}

This was an interventional case series in which two eyes of two patients with resistant fungal keratitis not responding to conventional teatment were subjected to intrastromal injection of the antifungal drug voriconazole. The diagnosis of fungal infection was observed on the basis of clinical evaluation, positive smear, and cultures of the fungus. Each patient was subjected to a detailed clinical evaluation that included documentation of ocular history and slit lamp biomicroscopy, visual acuity testing,

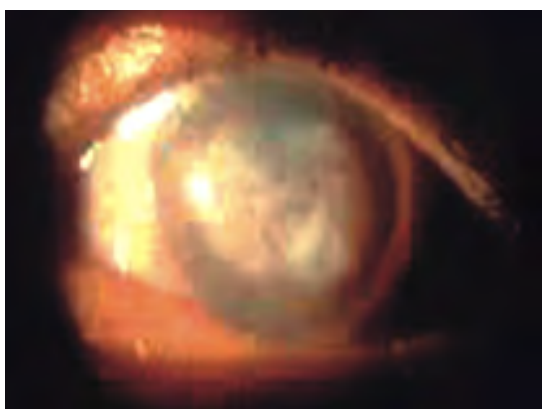

Fig. 1. Fungal ulcer (second case) at time of diagnosis.

Correspondence: Dr. P.K. Singh, B 33 Subhash Enclave, Airforce Area, Jodhpur, Rajasthan, India. E-mail: singhpunit12@gmail.com 
conventional topical therapy including drops of $5 \%$ natamycin and itraconazole every two hours, $1 \%$ homatropine (Fig. 1) .Corneal scrapings were obtained under topical anesthesia and were sent for potassium hydroxide $(\mathrm{KOH})$ wet-mount preparation, gram smear. Topical antifungal therapy was started including 5\% natamycin drops and itraconazole drops every two hours as soon as the fungus was identified by $\mathrm{KOH}$ wet-mount preparation and gram smear. When the infection showed signs of worsening, the patients were started on topical voriconazole $1 \%$ eye drops. If no response to this combined therapy was observed after 15 days, an antifungal drug was injected intrastromally perilesional around the fungal abscess.

Method of intrastromal injection: Two cases of fungal keratitis involving deep corneal stroma unresponsive to topical antifungal therapy underwent intrastromal injection of voriconazole $0.5 \mathrm{mg} / \mathrm{ml}$ or $50 \mu \mathrm{g} / 0.1 \mathrm{ml}$.

Availability: Voriconazole (Vfend, Pfizer Inc, New York, USA) is available as $200 \mathrm{mg}$ of lyophilized white powder in a glass vial. The preparation is used intravenously for systemic fungal infections and also for the preparation of voriconazole eye drops $1 \%$ (off label). Nowadays a preparation designed for topical use of Voriconazole is available as a $30 \mathrm{mg}$ vial (Vzole, Aurolab, Madurai, India), but we avoided using the preparation for intrastromal injections as it contains the preservative benzalkonium chloride. However, it may be used for topical application.

Intrastromal injection preparation: Add $20 \mathrm{ml}$ of ringer lactate to the vial (injection voriconazole for I.V.) for a concentration of $10 \mathrm{mg} / \mathrm{ml}$. Take $1 \mathrm{ml}$ of this preparation and furthur dilute to $20 \mathrm{ml}$. Now the concentration is $0.5 \mathrm{mg} / \mathrm{ml}$ or $50 \mu \mathrm{g} / \mathrm{ml}$.

Method of injection: Peribulbar anesthesia was given. Multiple injections were given around the abscess in the circumference of the lesion. The intention is to form a drug depot in the middle part of corneal stroma. The needle was injected from the uninvolved area to reach just adjacent to the abscess at the junction of the middle and anterior one third (mid-stromal level), in each case with the bevel down. Only a single sitting of intrastromal injections was given in both patients. No injections were repeated.

Table 1

\begin{tabular}{|l|l|l|l|l|}
\hline Age/sex & $\begin{array}{l}\text { Initial size of } \\
\text { abcess }\end{array}$ & $\begin{array}{l}\text { Species } \\
\text { identified }\end{array}$ & $\begin{array}{l}\text { Initial visual } \\
\text { acuity }\end{array}$ & $\begin{array}{l}\text { VA after 4 } \\
\text { weeks of } \\
\text { injection }\end{array}$ \\
\hline $1.35 / \mathrm{f}$ & $6.5 \times 4.5 \mathrm{~mm}$ & $\begin{array}{l}\text { Fusarium } \\
\text { species }\end{array}$ & $\begin{array}{l}\mathrm{PL}+\mathrm{PR} \\
\text { accurate only }\end{array}$ & $3 / 60$ \\
\hline $2.28 / \mathrm{f}$ & $3.5 \times 3.5 \mathrm{~mm}$ & $\begin{array}{l}\text { Aspergillus } \\
\text { species }\end{array}$ & CFCF & $3 / 60$ \\
\hline
\end{tabular}

\section{Results}

The total volume of drug injected intrastromally ranged from $0.05 \mathrm{ml}$ to $0.10 \mathrm{ml}$. In both the patients the procedure was performed satisfactorally and no intraor post-complications were observed. At each site of the injection hydration of the cornea was observed which was similar to the picture of hydrosealing a 
phaco-incision and is suggestive of the spread of the drug in the respective area. The hydration cleared in a few hours. The patient's smears were positive for fungus and on cultures, Fusarium species was identified in one eye and Aspergillus species also in one eye. After the intrastromal injections, both patients were continued on prescribed topical antifungal therapy. Patients were examined every day and response to the therapy was recorded, as well as visual acuity (BCVA) and measurement of size of abscess, using a slit-lamp. The patients were continued on topical antifungal therapy for at least one week after the complete resolution of infection. The infection was considered treated when there was complete healing of the epithelial defect with resolution of corneal abscess and scar formation.

The infection resolved completely in both eyes after the intrastromal injection of voriconazole over a period of 15 to 20 days (Figs. 2, 3) Only a mild improvement in visual acuity was seen in the cases attributable to involvement of the abscess and the resultant scar in the central cornea.

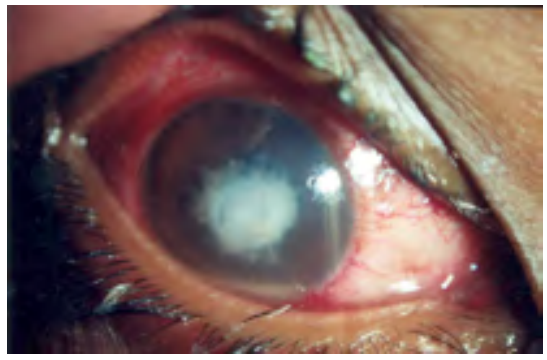

Fig. 2. Ulcer (first case) four weeks after intrastromal voriconazole.

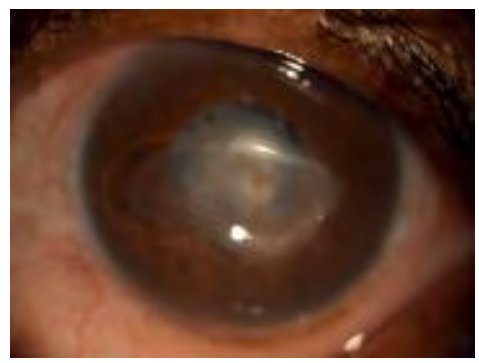

Fig. 3. After four weeks of injection (first case).

\section{Discussion}

Voriconazole, a derivative of fluconazole, is a new triazole antifungal agent. Like other triazoles, it inhibits cytochrome P450 demethylase, which is essential for the synthesis of ergosterol. It is hypothesized that this adversely affects the permeability of the fungal cell membrane. Voriconazole has excellent oral bio-availability and a broad spectrum of activity. Therapeutic aqueous and vitreous levels are achieved after oral administration of voriconazole.Voriconazole showed lower MICs compared to other antifungal agents when tested against five corneal isolates of Scedosporium apiospermum, and it had the best in- vitro susceptibility profile for 34 common fungal pathogens compared to other antifungal agents. Voriconazole has been shown to be highly effective against filamentous organisms and is more potent in invasive aspergillosis than amphotericin B. Voriconazole is also effective against candida chorioretinitis. Reports indicates that $1 \%$ voriconazole is an effective treatment for Fusarium and Aspergillus spp. and unknown fungal keratitis and candida as well.

This is particularly true for mycotic keratitis because none of the present-day antifungal agents can optimally penetrate the deeper layers of the cornea. To 
overcome these problems, modalities of targeted drug delivery are being evaluated. Similar attempts of site-directed drug deposit have been made in posterior segment pathologies in the form of intravitreal injections and posterior Sub-tenon injections of drugs. Intrastromal injections of amphotericin $B$ have been used previously to treat recalcitrant mycotic keratitis. ${ }^{7}$ We used voriconazole because previous experiences with it in ocular infections, using both topical and systemic routes, have been promising. Furthermore, voriconazole has optimal activity against fungi that are resistant to amphotericin $B$ and itraconazole and has a good safety profile. The intrastromal injections of voriconazole helped in early and complete resolution of the ulcers . Similar rewarding results were reported by Prakash et al. in series of three patients with resistant deep fungal karatitis subjected to intrastromal injections of voriconazole ${ }^{5}$ and Siatiri et al. in a study comaring intrastromal voriconazole with topical voriconazole $1 \%$ eye drops. ${ }^{6}$ However, we used oral voriconazole in neither of our patients and continued topical natamycin $5 \%$, topical itraconazole and topical Voriconazole 1\% eye drops before and after the intrastromal injection. Intrastromal injections were used only to supplement topical therapy and not as a replacement of conventional therapy.

In conclusion, intrastromal voriconazole is a new, promising supplemental therapy for fungal keratitis refractory to standard antifungal agents. Nevertheless, more clinical trials will be necessary to investigate the effectiveness of intrastromal voriconazole.

\section{References}

1. Kuriakose $T$, Kothari $M$, Paul $P$, Jacob $P$, et al. Intracameral amphotericin B injection in the management of deep keratomycosis. Cornea 2002;21:653-656.

2. Yoon KC, Jeong IY, Im SK, et al. Therapeutic effect of intracameral amphotericin B injection in the treatment of fungal keratitis. Cornea 2007;26:814-818.

3. Yilmaz S, Ture M, Maden A. Efficacy of intracameral amphotericin B injection in the management of refractory keratomycosis and endophthalmitis. Cornea 2007;26:398-402.

4. Freda R. Use of oral voriconazole as adjunctive treatment of severe corneal fungal infection: case report. Arq Bras Oftalmol 2006;69:431-434.

5. Prakash G, Sharma N,Goel M, et al. Evaluation of Intrastromal Injection of Voriconazole as a Therapeutic Adjunctive for the Management of Deep Recalcitrant Fungal Keratitis. Am J Ophthalmol 2008;146:56-59.

6. Siatiri H, Daneshgar F, Siatiri N, et al. The Effects of Intrastromal Voriconazole Injection and Topical Voriconazole in the Treatment of Recalcitrant Fusarium Keratitis. Cornea 2011;30:872-875.

7. Ghate D, Edelhauser HF. Ocular drug delivery. Expert Opin Drug Deliv 2006;3:275-287. 